\title{
Multifractal Analysis for Soft Fault Feature Extraction of Nonlinear Analog Circuits
}

\author{
Xinmiao Lu, Hong Zhao, Haijun Lin, and Qiong Wu \\ The Higher Educational Key Laboratory for Measuring \& Control Technology and Instrumentations of Heilongjiang Province, \\ Harbin University of Science and Technology, Harbin 150080, China
}

Correspondence should be addressed to Xinmiao Lu; lvxinmiao0611@126.com

Received 1 March 2016; Accepted 20 April 2016

Academic Editor: Anna Vila

Copyright (C) 2016 Xinmiao Lu et al. This is an open access article distributed under the Creative Commons Attribution License, which permits unrestricted use, distribution, and reproduction in any medium, provided the original work is properly cited.

\begin{abstract}
Aiming at the nonstationarity and nonlinearity of soft fault signals of nonlinear analog circuits, the use of multifractal detrended fluctuation analysis can effectively reveal the dynamic behavior hidden in multiscale nonstationary signals. This paper adopts a new method that uses multifractal detrended fluctuation analysis to calculate the multifractal singularity spectrum of soft fault signals of nonlinear analog circuits. Moreover, this method endows the parameters of the spectrum with definite physical meanings including width, maximum singular index, minimum singular index, and corresponding singularity index of the extreme point. Therefore, this method can be applied to characterize the internal dynamic mechanism of the soft fault signals of nonlinear analog circuits, making it suitable for the feature extraction of fault circuits. All multifractal feature parameters can be organized into a feature set, which will be then input to a support vector machine, and fault detection for the nonlinear analog circuit can be conducted via the support vector machine.
\end{abstract}

\section{Introduction}

The testing and fault diagnosis of nonlinear analog circuits have always been a hot research topic in the field of circuit testing. Although many important results have been achieved, the study of nonlinear systems is still in the stage of approximation process and numerical calculation. System modeling analysis, diagnosis theory, and its practicality all await further study. The nature of fault diagnosis of nonlinear analog circuits is pattern recognition, and the key to diagnosis is to construct fault features that reflect the nature of the tested circuit. Different approaches can be used to describe nonlinear analog circuits, including the Volterra series method, Wiener series method, and wavelet analysis-based feature extraction method of recent years.

The Volterra series can more accurately establish common fault models of nonlinear analog circuits. However, owing to the problem of dimensionality involved in using the Volterra series, in practice, only the low-order finite term can be taken. Furthermore, for some nonanalytic nonlinear systems, Volterra series expansion cannot be used. In addition, the terms in the Volterra series are not orthogonal to each other, making it difficult to extract the fault features of the circuit to be diagnosed, which reduces the effect of the fault diagnosis [1-3].

Lin et al. [4] solved this problem using the Wiener series. Gaussian white noise excitation is applied to a nonlinear system with unknown characteristic parameters, and an orthogonal functional series expansion is calculated for its response in order to obtain a set of functions that characterize system features. A time-correlation function is used to estimate the correlation function of each order to solve the Wiener kernels of the system, and a BP neural network is used to realize fault diagnosis. However, this method must be improved to obtain the Wiener kernels of the tested circuit more accurately and more efficiently [5].

The feature extraction theory based on wavelet analysis is the most utilized theory in the fault diagnosis of analog circuits. For the fault diagnosis of analog circuits, the biggest advantage of wavelet analysis and artificial neural networks is that the time-domain localized features are excellent, high frequencies and low frequencies are separated, the system is insensitive to circuit noise, and the noise removal ability is satisfactory, especially when there are many nonlinear 
components in the tested analog circuit [6-8]. When the ambient noise is particularly high, the frequency separation feature of wavelet analysis can function effectively, greatly reducing the difficulty of extracting circuit fault features. However, this method also requires a great amount of experimental data in order to form an accurate classification of the fault phenomena [9-11].

Chaotic dynamics and fractal theory developed in recent years provide a good direction for the study of nonlinear systems. Fractal theory is not only at the leading edge and an important branch of nonlinear science, but also an emerging transdisciplinary science. The use of the fractal method can accurately extract characteristics of the nonlinear signal. The majority of researchers use a single fractal to represent the overall irregular performance of signals. Zhang et al. [12] used the wavelet fractal method to perform a fault diagnosis of a three-phase bridge rectifier circuit. Mao et al. [13] also achieved satisfactory results by using analog circuit fault diagnosis based on a support vector machine (SVM) and fractal characteristics.

Peng et al. [14] proposed a detrended fluctuation analysis (DFA) that analyzes power-law functions; this analysis can effectively characterize the long-range correlation of the nonlinear time series of complex dynamic systems. However, the DFA method uses only a second-order wave function to analyze the scaling behavior of a sequence of events, obtaining only a single scaling exponent. Therefore, this method is only suitable for nonstationary time series with single scaling behavior (also known as a single fractal), which lacks a description of local singular characteristics. "Multifractal" not only describes the irregularity of signals as a whole but also more finely reflects the description of the local characteristics of the signal. Multifractal depicts the local scaling characteristics of the fractal subsets of different scales and scaling exponents distributed over the subsets.

Kantelhardt et al. [15] not only extended a onedimensional fractal DFA but also proposed an MF-DFA method by combining the one-dimensional fractal DFA with the standard partition function-based multifractal formalism. This method can effectively eliminate the interference trends and fluctuation scale of time series measurement, thus effectively overcoming the defects in DFA methods when analyzing multiscale multifractal nonstationary time series. In addition, this method can more accurately estimate the multifractal spectrum.

This paper introduces the MF-DFA method into nonlinear analog circuits and applies the MF-DFA method to analyze the collected signals for fault diagnosis based on the characteristic parameters of the multifractal spectrum of the fault signals.

\section{Estimating Multifractal Spectrum Using the MF-DFA Method}

Assume that the time series to be detected has a signal length $N\left\{x_{t}, t=1,2, \ldots, N\right\}$. The DFA method is conducted as follows.
Step 1. Calculate the average value of the original time series. Sum the difference of the terms between the given time series $x_{t}$ and the average value, producing a new contour sequence $y(i)$ :

$$
y(i)=\sum_{t=1}^{i}\left(x_{t}-\bar{x}\right) \quad i=1,2, \ldots, N,
$$

where $\bar{x}=(1 / N) \sum_{t=1}^{N} x_{t}$ and $\bar{x}$ is the average value of the time sequence $\left\{x_{t}, t=1,2, \ldots, N\right\}$.

Step 2. Divide the new sequence $y(i)$ obtained from the transform into $m$ nonoverlapping subintervals of equal length $s$ so that $m=\operatorname{int}(N / s)$, which is a rounding process for $N / s$. Because $N$ may not be a multiple of $s$, to avoid the loss of information from the original time series, the new sequence $y(i)$ is also divided in the same way, so that all figures in series $y(i)$ are in the calculation. Finally, the $2 m$ subintervals of equal set length $s$ can be obtained.

Step 3. Remove the fluctuation trend of each small section using the least squares method. Assume that $y_{v}(i)$ is the $m$ th order of the least squares fitting polynomial of the $v(v=$ $1,2, \ldots, 2 m)$ th small interval. The function is the $k$ th-order polynomial fitting of the $v$ th subinterval for $s$ observations, and the fitting equation obtained is as follows:

$$
\begin{aligned}
y_{v}(i)=a_{0}+a_{1} i+a_{2} i^{2}+\cdots & +a_{k} i^{k} \\
& \quad i=1,2, \ldots, s ; k=1,2, \ldots
\end{aligned}
$$

In the formula, $a_{k}$ is the fitting polynomial, and $k$ is the order of the fitting polynomial.

Step 4. Eliminate the trend within each subinterval, and calculate the mean square error $F_{v}^{2}(s)$.

When $v=1,2, \ldots, N_{s}$, there is

$$
F_{v}^{2}(s)=\frac{1}{s} \sum_{i=1}^{s}\left\{Y[(v-1) s+i]-y_{0}(i)\right\}^{2} .
$$

When $v=N_{s}+1, N_{s}+2, \ldots, 2 N_{s}$, there is

$$
F_{v}^{2}(s)=\frac{1}{s} \sum_{i=1}^{s}\left\{Y\left[N-\left(v-N_{s}\right) s+i\right]-y_{v}(i)\right\}^{2} .
$$

Step 5. For the $2 N_{s}$ detrended intervals, take the average value of $F_{v}^{2}(s)$ to obtain the $q$ th wave function:

$$
\begin{aligned}
& F_{q}(s)=\left\{\frac{1}{2 N} \sum_{v=1}^{2 N_{s}}\left[F_{v}^{2}(s)\right]^{q / 2}\right\}^{1 / q} \quad q \neq 0, \\
& F_{q}(s)=\exp \left\{\frac{1}{4 N} \sum_{v=1}^{2 N_{s}} \ln \left[F_{v}^{2}(s)\right]\right\} \quad q=0 .
\end{aligned}
$$

When $q=2$, MF-DFA degenerates into DFA; different $q$ values describe the impact on $F_{q}(s)$ from different degrees of fluctuation. The mean square error $F_{v}^{2}(s)$ can eliminate the trend of each small section using the polynomial fitting of each subinterval, which is more beneficial for the singularity recognition of local fluctuation. 
Step 6. By analyzing the relationship in the double logarithmic plot $F_{q}(s) \sim s^{h(q)}$, the scaling exponent of the wave function $h(q)$ can be determined, so a power-law relationship exists:

$$
F_{q}(s) \propto s^{h(q)} .
$$

Each $s$ corresponds to a wave function $F_{q}(s)$. The wave function value $F_{q}(s)$ is an increasing function of $s$. Draw the relationship plot of $\lg F_{q}(s)$ with respect to $\lg s$, and the slope obtained from the linear fitting of the least squares method is $h(q)$, also known as the generalized Hurst exponent. When $h(q)$ is constant, the time series appears as a single fractal. When $h(q)=0.5$, the time series appears either without a correlation or with a short correlation. When $h(q)$ has a nonlinear relationship with $q$, the time series appears to have multifractal characteristics.

Step 7. Between the generalized Hurst exponent $h(q)$ from the discrete time series and the mass index $\tau(q)$ from classic multifractal theory, there exists

$$
\tau(q)=q h(q)-1
$$

In this way, the relationship between the generalized Hurst exponent $h(q)$ and the scaling index $\tau(q)$ from the partition function-based method can be obtained. With the Legendre transform from statistical physics,

$$
\begin{aligned}
& \alpha(q)=\frac{d \tau(q)}{d q}, \\
& f(\alpha)=q \alpha(q)-\tau(q),
\end{aligned}
$$

the relationship between the multifractal spectral index $\alpha$, $f(\alpha)$, and the generalized Hurst exponent $h(q)$ is obtained:

$$
\begin{aligned}
& \alpha(q)=h(q)+\frac{q d h(q)}{d q}, \\
& f(\alpha)=q[\alpha(q)-h(q)]+1 .
\end{aligned}
$$

\section{Feature Extraction of Multifractal Spectrum}

The multifractal spectrum obtained from the MF-DFA method is a set of parameters that can finely portray the dynamic behavior of a multifractal time series. This paper will adopt six types of commonly used multifractal spectrum parameters as the fault characteristic values of soft fault signals of a nonlinear analog circuit. The parameters of multifractal singularity spectrum $\alpha \sim f(\alpha)$ are as follows:

(1) The width of the multifractal singularity spectrum $\Delta \alpha=\alpha_{\max }-\alpha_{\min }: \alpha_{\min }$ corresponds to the minimum probability subset, $\alpha_{\max }$ corresponds to the maximum probability subset, and $\Delta \alpha$ represents the multifractal strength of a time series, a greater value of which would mean a more uneven distribution of probability measure of the time series and a more intense change in the signals. $\Delta \alpha$ can describe the degree of uneven distribution of circuit detection signals.

(2) The dimensional difference $\Delta f=f\left(\alpha_{\min }\right)-f\left(\alpha_{\max }\right)$ : $f\left(\alpha_{\text {min }}\right)$ represents the fractal dimension of the maximum
TABLE 1: Fault mode setting.

\begin{tabular}{lccc}
\hline Fault codes & Fault states & Fault codes & Fault states \\
\hline 0 & Normal & 10 & $R_{11}+30 \%$ \\
1 & $R_{1}+30 \%$ & 11 & $C_{1}+30 \%$ \\
2 & $R_{1}-30 \%$ & 12 & $C_{1}-30 \%$ \\
3 & $R_{2}+30 \%$ & 13 & $C_{2}+30 \%$ \\
4 & $R_{2}-30 \%$ & 14 & $C_{2}-30 \%$ \\
5 & $R_{4}-30 \%$ & 15 & $R_{5}-50 \%$ \\
6 & $R_{6}+30 \%$ & 16 & $R_{9}+50 \%$ \\
7 & $R_{7}-30 \%$ & 17 & $R_{\mathrm{OA1}}+50 \%$ \\
8 & $R_{8}+30 \%$ & 18 & $R_{\mathrm{OA} 3}-50 \%$ \\
9 & $R_{10}-30 \%$ & 19 & $R_{\mathrm{OA} 5}+50 \%$ \\
\hline
\end{tabular}

fluctuation point set, and $f\left(\alpha_{\max }\right)$ represents the fractal dimension of the minimum fluctuation point set. $\Delta f$ reflects the frequency ratio of the maximum fluctuation to the minimum fluctuation. The value of $\Delta f$ reflects the proportion of the number of most stable subsets and most volatile subsets in the fluctuation in the circuit monitor signals.

\section{Experimental Simulation and Analysis}

The MF-DFA method can be used to evaluate the fault feature extraction based on six parameters of the multifractal spectrum. To assess the performance of MF-DFA for soft fault signal analysis of nonlinear analog circuits, an experimental fault diagnosis simulation of chaotic circuits previously described in the literature [16] is conducted. The structure and parameters of the circuit are shown in Figure 1.

In this experiment, the selected excitation frequency is $0.155159 \mathrm{~Hz}$, the amplitude is $0.7414148 \mathrm{~V}$, and the twodimensional phase plane of node voltages $U_{1}$ and $U_{2}$ at this time is shown in Figure 2.

The normal tolerance of resistor components and capacitor components is $5 \%$ of their nominal value. In this test, a drift of plus or minus $30 \%$ and $50 \%$ of the nominal value is set as a fault condition. Only the signals at output node $U_{1}$ are collected and analyzed, while the other measureable nodes are not included in this discussion. The fault mode setting is shown in Table 1. A total of 20 types of parametric fault combinations are tested in the experiment, in which the fault state corresponding to each fault uses a representation of “(element 1 AND element 2) $\pm 30 \%$," meaning that each element in parentheses has a drift of either positive or negative $30 \%$. For instance, " $\left(R_{1}\right.$ AND $\left.R_{2}\right)+30 \%$ " indicates a fault type in which resistors $R_{1}$ and $R_{2}$ each have a $30 \% \mathrm{drift}$ from their nominal values. " $R_{\mathrm{OA} i}$ " indicates equivalent input impedance of amplifier. PSpice 9.1 software is used to simulate the circuits in a variety of fault states and normal states 50 times each and to collect 2000 voltage signals in the $[0,30]-\mathrm{s}$ time period from output node $U_{1}$ as the original features of each fault state.

In this analysis, signals from faults 12 and 16 are used as examples. The signal time series waveform collected at node $U_{1}$ is shown in Figure 3. As can be seen from the figure, the time series waveforms from fault 12 and fault 16 are fairly close 


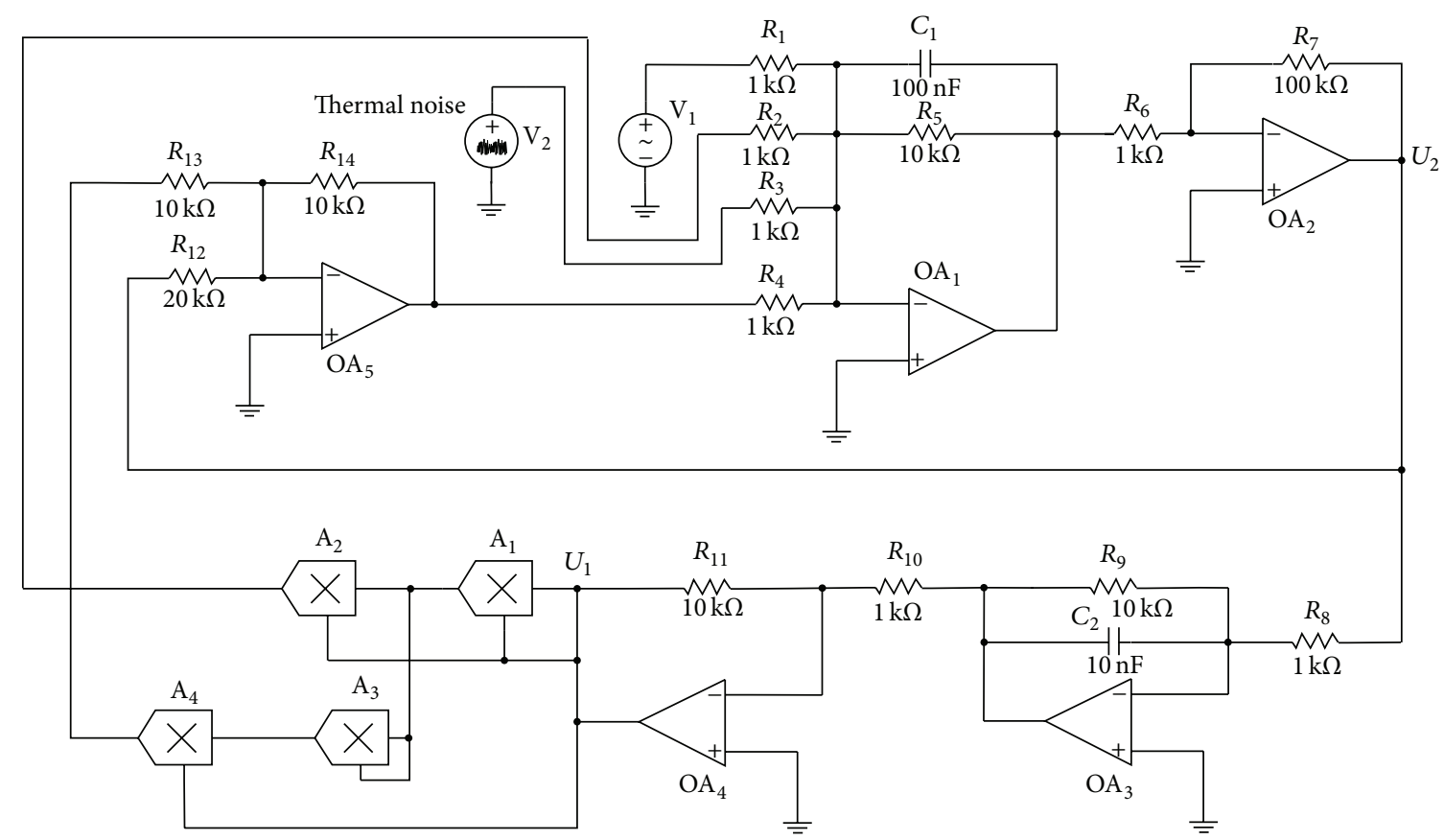

FIgURe 1: Chaotic circuits system.

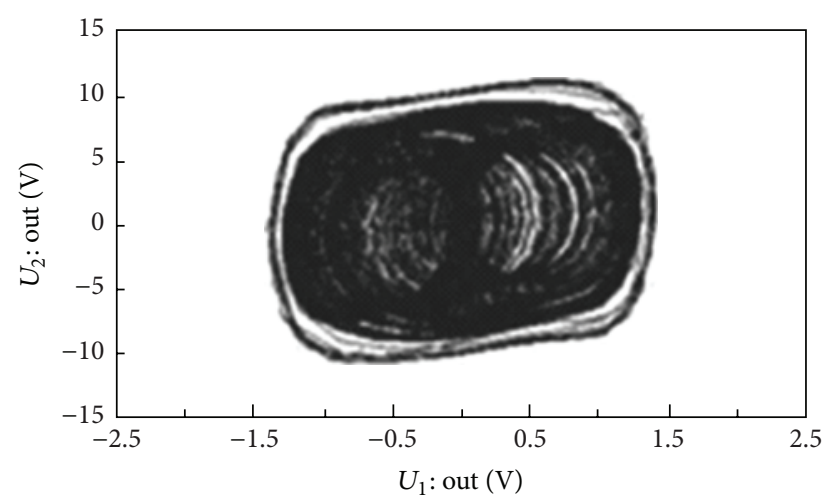

FIGURE 2: Two-dimensional phase plane of node voltages $U_{1}$ and $U_{2}$.

to each other, and it is difficult to distinguish fault 12 and fault 16 from the time series plot. The MF-DFA is used to process these signals.

Figure 4 shows the function diagram of $h(q) \sim q$ of two types of faults. It can be derived from the figure that in both types of faults there is a nonlinear relationship between $h(q)$ and $q$, indicating that both faults have multifractal characteristics. With the $q$ value fixed, the relationship between the detrended fluctuation logarithmic function of two faults and different logarithmic scale $\lg F_{q}(s) \sim \lg s$ is shown in Figures 5 and 6.

The Legendre transform can be used to obtain the numerical solution of $\alpha$ and $f(\alpha)$. The multifractal spectrum $f(\alpha)$ of two types of faults is shown in Figure 7. Six parameter values $\left[\alpha_{\min }, \alpha_{\max }, \Delta \alpha, f\left(\alpha_{\min }\right), f\left(\alpha_{\max }\right)\right.$, and $\left.\Delta f\right]$ of fault 12

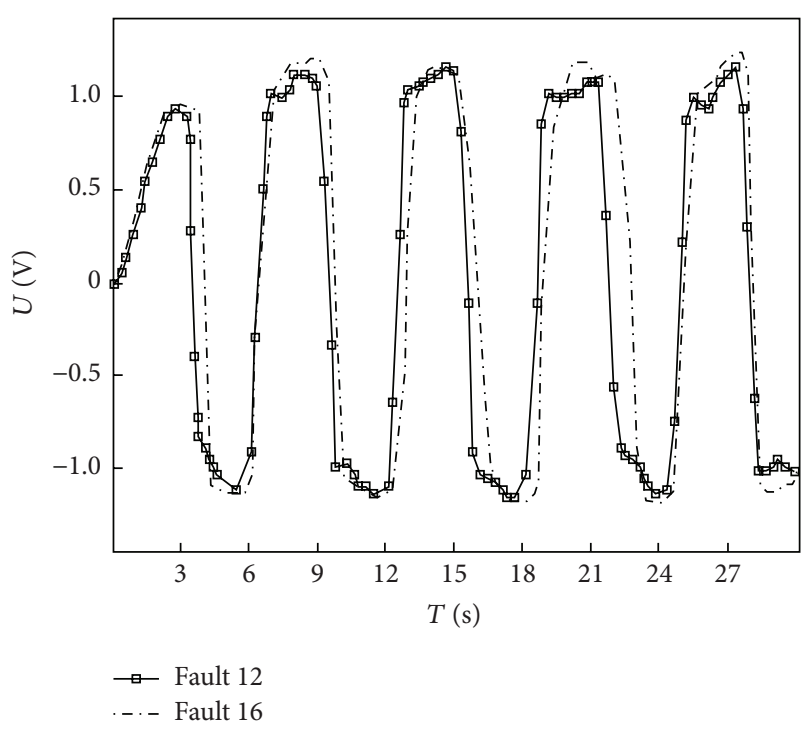

Figure 3: Time series waveforms from fault 12 and fault 16.

and fault 16 can be obtained from the figure. Using these six commonly used multifractal spectrum parameters as fault characteristic values of soft fault signals of the nonlinear analog circuit, the features of circuits under different states can be easily distinguished.

Using the MF-DFA for collected data from 20 types of faults, different multifractal singularity spectrum graphs can be obtained (hereby omitted), thereby extracting different fault characteristic parameters from different multifractal singularity spectrum graphs. Eventually, this constitutes 20 


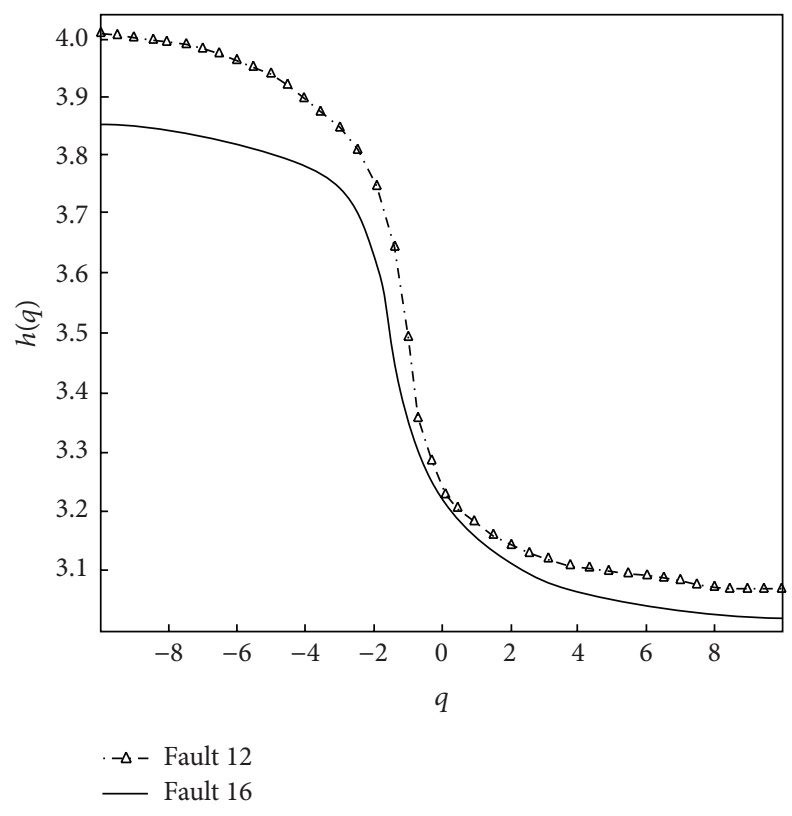

FIGURE $4: h(q) \sim q$ of fault 12 and fault 16 .

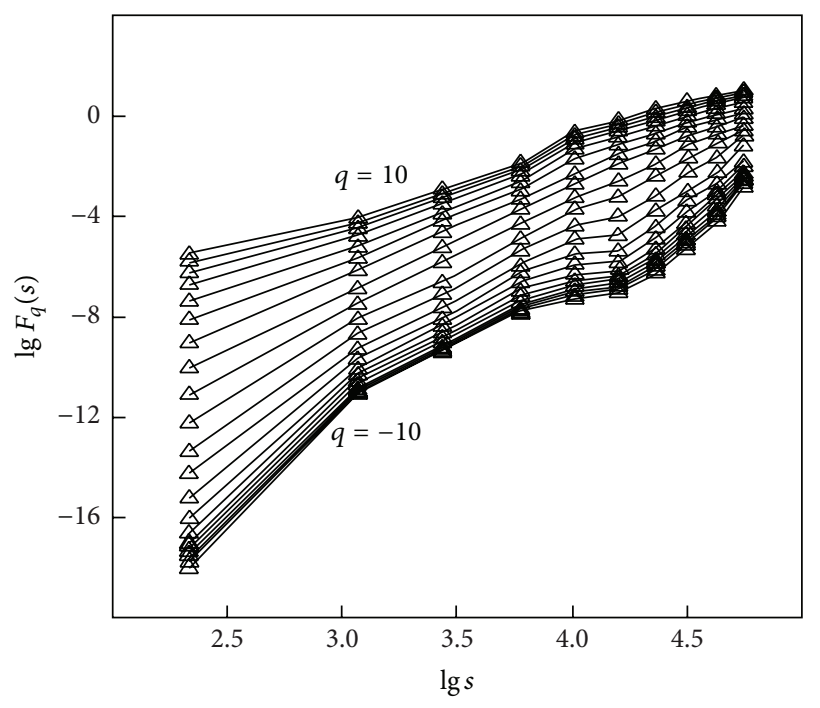

Figure 5: $\lg F_{q}(s) \sim \lg s$ of fault 12.

groups of fault characteristic parameters, as shown in Table 2, where different fault conditions have different multifractal characteristic parameters. Using the MF-DFA, we can effectively extract the fault features to distinguish different states.

To verify the effectiveness of the approach, the extracted multifractal feature set was inputted into the support vector machine for the experiment of fault classification. Among the 50 feature samples for each fault mode, 30 feature samples were randomly selected to train the support vector machine; the rest of the feature samples were used to test the support vector machine. The accuracy of the fault diagnosis results was as high as $93 \%$, thus proving the effectiveness of the multifractal analysis for soft fault feature extraction of nonlinear analog circuits.

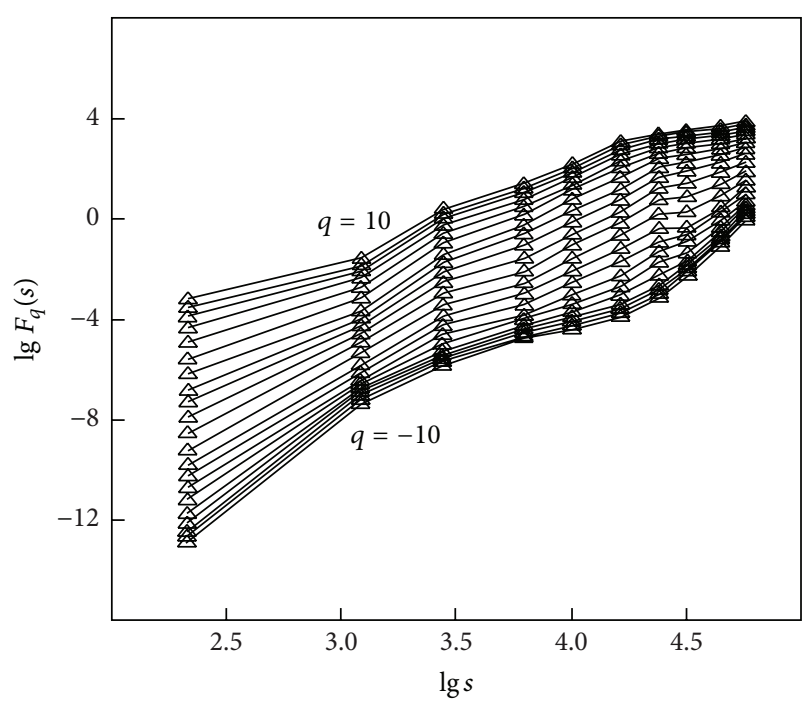

FIGURE 6: $\lg F_{q}(s) \sim \lg s$ of fault 16.

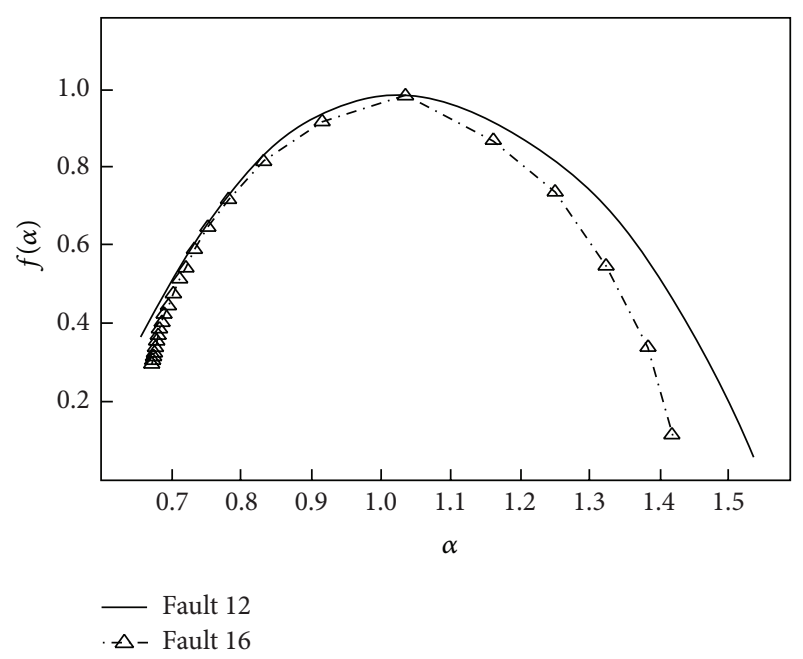

FIGURE 7: $f(\alpha) \sim \alpha$ of fault 12 and fault 16.

\section{Conclusion}

The fractal theory can effectively analyze the inherent nature and characteristics of nonlinear signals and quantitatively describe the irregularities of fractal characteristics and signals of nonlinear systems. The soft fault signals of an analog circuit are nonstationary and nonlinear. This paper adopts MF-DFA to analyze the soft fault signals of a nonlinear analog circuit. Based on the multifractal characteristic parameters of different fault modes, this method is verified by a simulation experiment using a chaotic circuit system. The multifractal feature parameters are inputted to the support vector machine for fault detection. The results confirmed that the method can effectively extract fault features and provide favorable conditions for the realization of analog circuit fault detection. 
TABLE 2: Multifractal characteristic parameters of fault signals.

\begin{tabular}{|c|c|c|c|c|c|c|}
\hline Fault codes & $\alpha_{\min }$ & $\alpha_{\max }$ & $\Delta \alpha$ & $f\left(\alpha_{\min }\right)$ & $f\left(\alpha_{\max }\right)$ & $\Delta f$ \\
\hline 0 & 0.651 & 1.726 & 1.075 & 0.227 & 0.019 & 0.208 \\
\hline 1 & 0.617 & 1.533 & 0.916 & 0.351 & -0.058 & 0.409 \\
\hline 2 & 0.669 & 1.835 & 1.166 & 0.272 & -0.121 & 0.393 \\
\hline 3 & 0.626 & 1.525 & 0.899 & 0.245 & -0.083 & 0.328 \\
\hline 4 & 0.630 & 1.517 & 0.887 & 0.236 & -0.117 & 0.353 \\
\hline 5 & 0.704 & 2.138 & 1.434 & 0.265 & -0.126 & 0.391 \\
\hline 6 & 0.688 & 1.841 & 1.153 & 0.263 & -0.113 & 0.376 \\
\hline 7 & 0.713 & 1.926 & 1.213 & 0.300 & 0.076 & 0.224 \\
\hline 8 & 0.666 & 1.701 & 1.035 & 0.473 & -0.143 & 0.616 \\
\hline 9 & 0.683 & 1.714 & 1.031 & 0.384 & -0.161 & 0.545 \\
\hline 10 & 0.721 & 1.644 & 0.923 & 0.359 & -0.157 & 0.516 \\
\hline 11 & 0.740 & 1.528 & 0.788 & 0.333 & 0.101 & 0.232 \\
\hline 12 & 0.642 & 1.532 & 0.890 & 0.363 & 0.056 & 0.307 \\
\hline 13 & 0.631 & 1.927 & 1.296 & 0.244 & -0.153 & 0.397 \\
\hline 14 & 0.680 & 1.707 & 1.027 & 0.259 & -0.150 & 0.409 \\
\hline 15 & 0.704 & 1.817 & 1.113 & 0.323 & 0.097 & 0.226 \\
\hline 16 & 0.667 & 1.423 & 0.756 & 0.310 & 0.116 & 0.194 \\
\hline 17 & 0.637 & 1.662 & 1.025 & 0.214 & 0.017 & 0.197 \\
\hline 18 & 0.671 & 1.804 & 1.133 & 0.229 & 0.025 & 0.204 \\
\hline 19 & 0.634 & 1.701 & 1.067 & 0.210 & 0.015 & 0.195 \\
\hline
\end{tabular}

\section{Competing Interests}

The authors declare that they have no competing interests.

\section{References}

[1] T. Wang and T. J. Brazil, "Volterra-mapping-based behavioral modeling of nonlinear circuits and systems for high frequencies," IEEE Transactions on Microwave Theory and Techniques, vol. 51, no. 5, pp. 1433-1440, 2003.

[2] H. Y. Yuan, T. L. Wang, and G. Y. Chen, "Fault diagnosis method in nonlinear analog circuit based on volterra frequency domain kernel and neural network," Chinese Journal of Scientific Instrument, vol. 28, no. 5, pp. 807-811, 2007.

[3] H. F. He, Y. C. Chen, Z. Yang, and H. Deng, "Fault diagnosis method of non-linear analog circuits based on Volterra series and SVM," in Proceedings of the 26th Chinese Control and Decision Conference (CCDC '14), pp. 3217-3222, Changsha, China, June 2014.

[4] H. J. Lin, L. Y. Zhang, D. Y. Ren, H. Kang, and G. Gu, "Fault diagnosis in nonlinear analog circuit based on Wiener kernel and BP neural network," Chinese Journal of Scientific Instrument, vol. 30, no. 9, pp. 1946-1949, 2009.

[5] M. Badoni, A. Singh, and B. Singh, "Comparative performance of wiener filter and adaptive least mean square-based control for power quality improvement," IEEE Transactions on Industrial Electronics, vol. 63, no. 5, pp. 3028-3037, 2016.

[6] Y.-H. Tan and J.-Z. Ye, "Wavelet method for fault diagnosis of analogue circuits," Journal of Electronics and Information Technology, vol. 28, no. 9, pp. 1748-1751, 2006.

[7] M. R. Liu, Y. G. He, G. F. Fang, X. Yin, and S.-z. Qi, "Application for fault diagnosis of analog circuits based on GA, wavelet and
NN," Journal of Hunan University(Natural Sciences), vol. 36, no. 3, pp. 40-44, 2009.

[8] S. X. Tang, H. J. Cai, and Z. G. Li, "Fault diagnosis fusion method for analog circuits based on wavelet and neural network," Journal of Central South University (Science and Technology), vol. 46, no. 1, pp. 127-134, 2015.

[9] X. Q. Liu and D. Z. Wang, "Wavelet neural networks based fault diagnosis of analog circuit," in Proceedings of the 24th Chinese Control and Decision Conference (CCDC '12), pp. 2234-2239, Taiyuan, China, May 2012.

[10] M. G. Dimopoulos, D. K. Papakostas, B. D. Vassios, and A. A. Hatzopoulos, "Wavelet analysis of current measurements for mixed-signal circuit testing," in Proceedings of the IEEE International Symposium on Circuits and Systems: Nano-Bio Circuit Fabrics and Systems (ISCAS '10), pp. 1923-1926, Paris, France, June 2010.

[11] W. Li, A. Monti, and F. Ponci, "Fault detection and classification in medium voltage Dc shipboard power systems with wavelets and artificial neural networks," IEEE Transactions on Instrumentation and Measurement, vol. 63, no. 11, pp. 2651-2665, 2014.

[12] H.-T. Zhang, Q. An, Z.-K. Hu, and Z.-W. Chen, "Fault detection wavelet fractal method of circuit of three-phase bridge rectifier," in Proceedings of the International Conference on Intelligent System Design and Engineering Application (ISDEA '10), vol. 1, pp. 725-729, Changsha, China, October 2010.

[13] X. B. Mao, L. H. Wang, and C. X. Li, "SVM classifier for analog fault diagnosis using fractal features," in Proceedings of the 2nd International Symposium on Intelligent Information Technology Application (IITA '08), pp. 553-557, Shanghai, China, December 2008.

[14] C.-K. Peng, S. Havlin, H. E. Stanley, and A. L. Goldberger, "Quantification of scaling exponents and crossover phenomena in nonstationary heartbeat time series," Chaos, vol. 5, no. 1, pp. 82-87, 1995. 
[15] J. W. Kantelhardt, S. A. Zschiegner, E. Koscielny-Bunde, S. Havlin, A. Bunde, and H. E. Stanley, "Multifractal detrended fluctuation analysis of nonstationary time series," Physica A: Statistical Mechanics and Its Applications, vol. 316, no. 1-4, pp. 87-114, 2002.

[16] W. J. Hu, Z. Z. Liu, and Z. H. Li, "Weak signal detection performance of improved Duffing circuit," Electric Machines and Control, vol. 9, no. 15, pp. 80-85, 2011. 


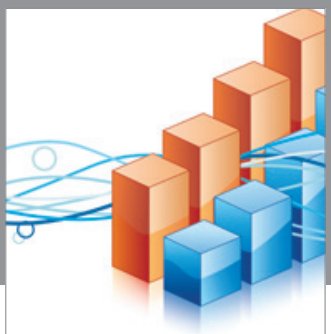

Advances in

Operations Research

vatem alat4

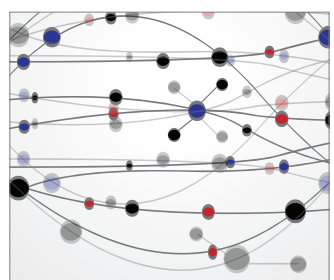

\section{The Scientific} World Journal
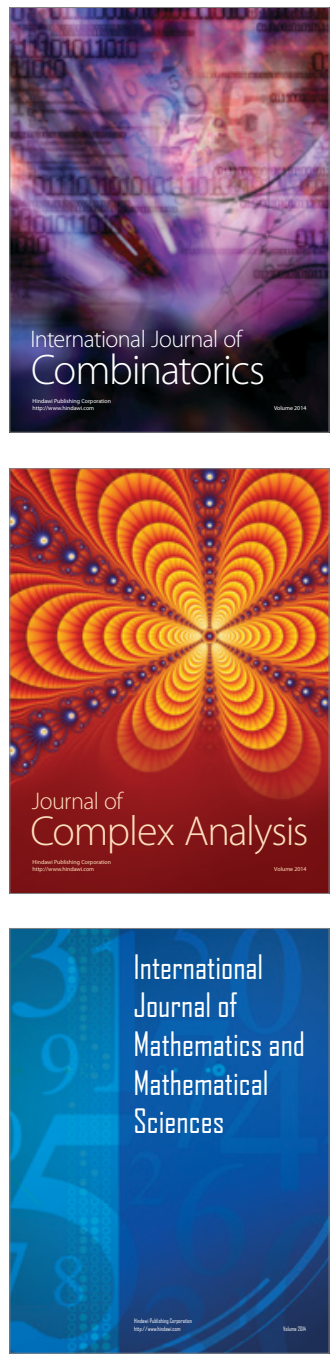
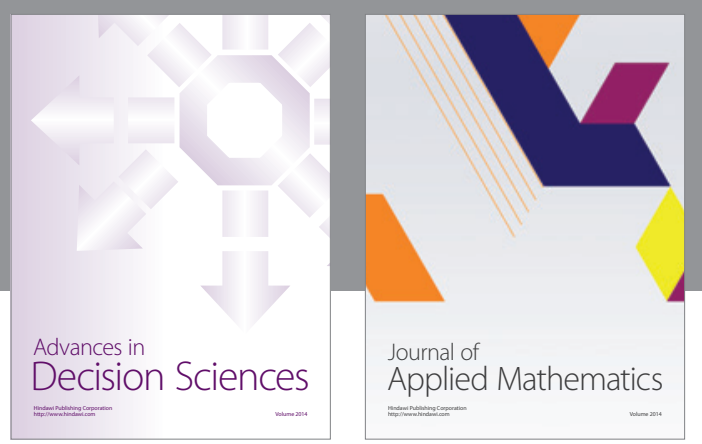

Algebra

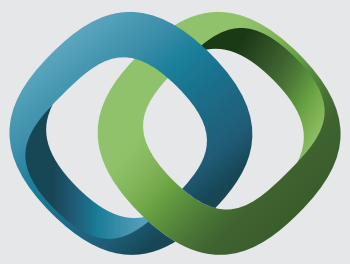

\section{Hindawi}

Submit your manuscripts at

http://www.hindawi.com
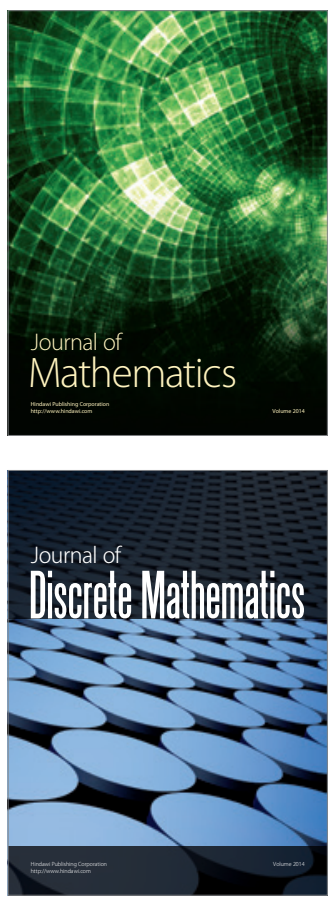

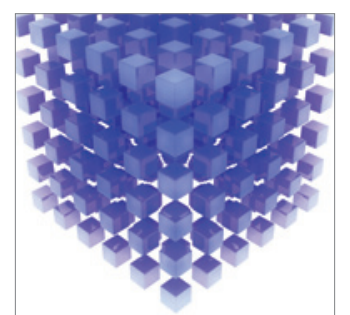

Mathematical Problems in Engineering
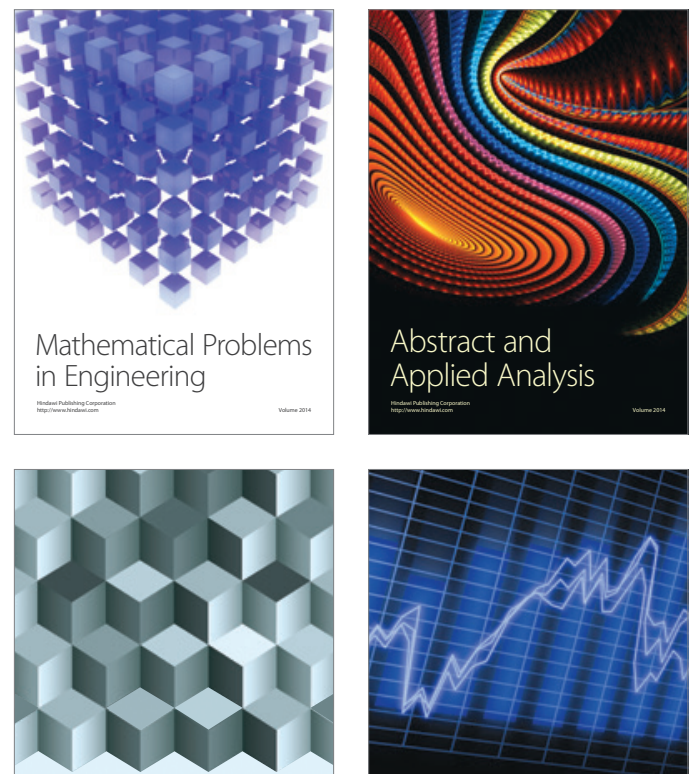

Journal of

Function Spaces

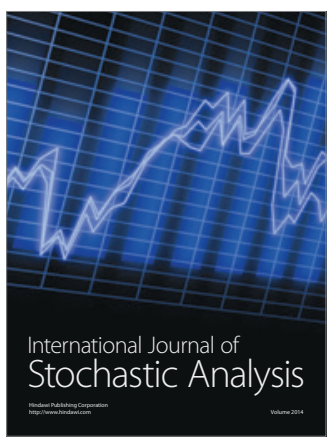

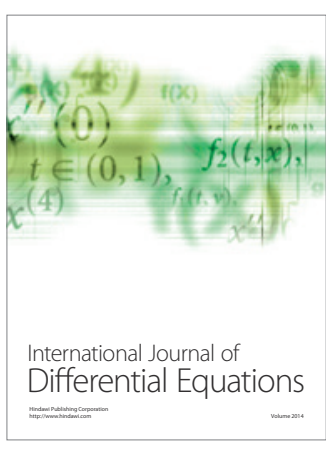
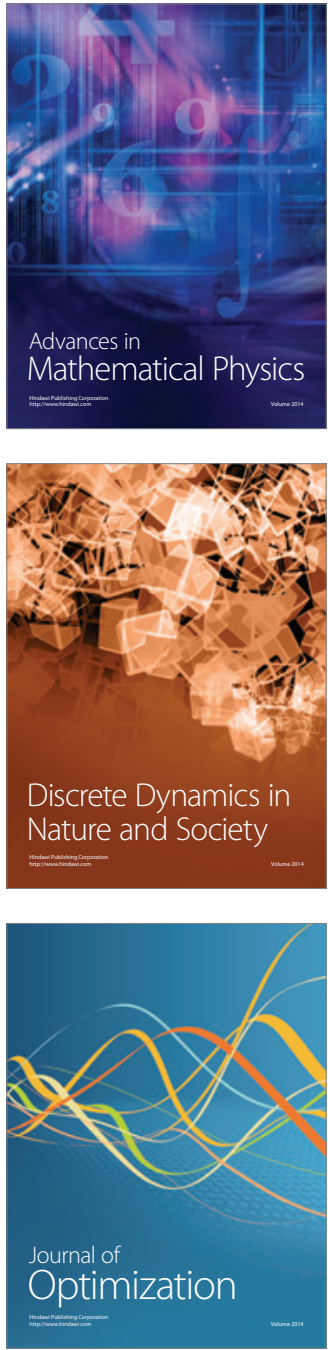